\title{
Correction
}

\section{Correction: Sequerra, Goyal et al., "NMDA Receptor Signaling Is Important for Neural Tube Formation and for Preventing Antiepileptic Drug-Induced Neural Tube Defects"}

In the article "NMDA Receptor Signaling Is Important for Neural Tube Formation and for Preventing Antiepileptic Drug-Induced Neural Tube Defects" by Eduardo B. Sequerra, Raman Goyal, Patricio A. Castro, Jacqueline B. Levin, and Laura N. Borodinsky, which appeared on pages 4762-4773 of the May 16, 2018 issue, an incorrect acknowledgment was listed. The authors apologize for the oversight. The corrected acknowledgments are as follows: "This work was supported by the Basil O'Connor Starter Scholar Research Award Grant 5-FY09-131 from the March of Dimes Foundation; a Klingenstein Foundation Award in Neuroscience; National Science Foundation Grant 1120796; National Institutes of Health-National Institute of Neurological Disorders and Stroke Grant R01-NS073055; and Shriners Hospital for Children Grants 86500-NCA, 85220-NCA, and 85300-NCA (to L.N.B.); by the Epilepsy Foundation and Shriners Hospital for Children postdoctoral fellowships (to E.B.S.); and by Fondecyt Inicio 11160562 (to P.A.C.). We thank Drs. Nicholas Spitzer and Andrew Hamilton for comments on the manuscript." This correction does not affect the conclusions of the paper. The acknowledgments have been corrected in the on-line PDF version.

DOI: $10.1523 / J N E U R O S C I .2632-18.2018$ 